\title{
La adaptación de los códigos de ética periodística europeos a Internet y las TIC
}

institucional.us.es/ambitos/

\section{Jesús Díaz-Campo}

Universidad Internacional de la Rioja

jesus.diaz@unir.net

\section{Francisco Segado-Boj}

Universidad Internacional de la Rioja

francisco.segado@unir.net

\section{Resumen}

El periodismo afronta nuevos problemas éticos debido al surgimiento y posterior consolidación de Internet y las Tecnologías de la Información y la Comunicación (TIC). En este artículo se analiza hasta qué punto se han adaptado los códigos de ética periodística para hacer frente a esta nueva realidad. Para ello se desarrollan

tres preguntas de investigación a través del análisis sistemático de 45 códigos europeos. Los resultados muestran que, de éstos, sólo ocho contienen referencias a Internet y a las TIC. Por último, se plantean varias propuestas para que sean incluidas en los códigos y ayuden a los periodistas a resolver los nuevos problemas

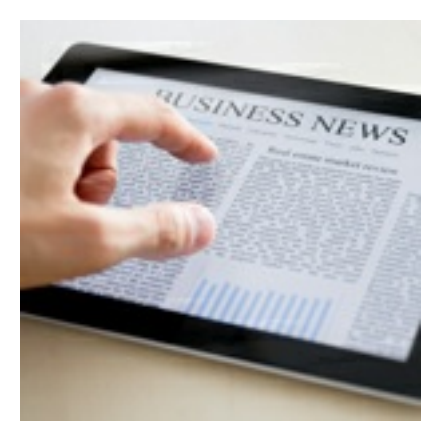

éticos.

\section{Palabras clave}

Códigos de ética periodística, periodismo digital, Internet, autorregulación, Europa.

Abstract

Journalism is facing new ethical issues because of the emergence of Internet and information and communication technologies (ICTs). In this paper, we examine how journalism codes of ethics have been updated to deal with this new reality. Three research questions are explored through systematic analysis of 45 codes from Europe. Results show that, of the 45 analyzed codes, only 8 of them include references to Internet and ICTs. Finally, we make some proposals to be included in the codes and to help journalists solving those new ethical issues.

\section{Keywords}

Journalism codes of ethics, online journalism, Internet, self-regulation, Europe.

\section{INTRODUCCIÓN (1)}

La eclosión de las Tecnologías de la Información y la Comunicación (TIC) y, en particular, de Internet, ha provocado una serie de transformaciones en el ámbito del periodismo. Estos cambios desembocan en el surgimiento de una serie de dilemas éticos muchos de los cuales no son en absoluto novedosos. Sin embargo, este nuevo ecosistema digital provoca que los periodistas, y el periodismo como profesión, se vean enfrentados a cuestiones morales particulares que se suman a los dilemas tradicionales.

De hecho, se puede afirmar que es el propio proceso periodístico el que se ha modificado de manera sustancial, a través de cambios que afectan sobre todo a la forma en la que el medio se relaciona con su público (Boler, 2008; Jenkins, 2006; López García, 2005; Schultz, 2000), de modo que éste último tiene la oportunidad de tomar parte en todas las fases de producción de la información (Bowman y Willis, 2003) hasta el punto de que siente el medio como un espacio propio (Lara, 2008). Ésta es una de las muchas y significativas 
transformaciones que han tenido lugar en el mundo del periodismo durante los últimos años. La interactividad, la hipertextualidad, la inmediatez o el multimedia son algunos de los elementos que caracterizan al periodismo digital y es evidente que cada uno de ellos genera nuevos debates éticos: Así por ejemplo, Evers (2001: 38) se cuestiona: "¿hasta qué punto es el propietario de un sitio web responsable legal y éticamente de lo que se publica en ese sitio?" (incluyendo, por ejemplo, comentarios anónimos). En esa misma línea cabría preguntarse: ¿es un sitio web responsable de que un enlace pueda conducir al usuario a ver contenido ofensivo? En definitiva, son muchos los nuevos retos a los que se enfrenta el periodismo digital, como por ejemplo la presencia de periodistas en las redes sociales, la abundancia de información sin verificar o el papel de la comunidad en la producción de noticias (McBride y Rosenstiel, 2013). La vertiente moral de todos ellos es innegable.

Por tanto, la cuestión de fondo que subyace en este panorama podría formularse de la siguiente manera: ¿Se pueden aplicar las mismas reglas y criterios éticos del periodismo tradicional al periodismo digital? Lo cierto es que como señalan Deuze y Yeshua (2001: 276) internet "da forma y redefine una serie de cuestiones éticas y morales a las que tienen que hacer frente los periodistas cuando trabajan en el entorno online o emplean fuentes que han encontrado en la red". Son muchos los autores (Bardoel y Haenens, 2004; Birsen, 2011; CruzÁlvarez y Suárez-Villegas, 2012; Evers, 2001; Demir, 2011; García Capilla, 2012; Holt y Von Krogh, 2010; Pavlik, 2001, Whitehouse, 2010, entre otros) que comparten el concepto de Deuze y Yeshua y coinciden en señalar que los nuevos medios de comunicación requieren una nueva ética, ya que los problemas a los que se tienen que enfrentar los periodistas que trabajan en medios digitales son diferentes de los que afrontan los que trabajan en medios tradicionales.

Por tanto, tal y como resaltan Hayes, Singer y Ceppos $(2007,275)$, en el nuevo ecosistema digital, "ya no es posible aceptar de modo indefinido e incondicional los presupuestos tradicionales sobre los roles y los valores periodísticos". En otras palabras, los nuevos conflictos éticos requieren nuevas normas éticas o, al menos, que los estándares éticos tradicionales sean reformulados.

Uno de los instrumentos con los que los periodistas se han enfrentado a cada nuevo desafío ético ha sido la autorregulación, que constituye según Evers $(2001,46)$ "la única vía posible para crear una serie de estándares para el periodismo online y de controlar que las normas morales son tenidas en cuenta". Siguiendo a Aznar (2005, 13-14) la autorregulación se caracteriza porque uno de sus objetivos es hacer efectiva la deontología de una determinada actividad o de contribuir a ello; y porque quienes la impulsan y le dan continuidad son las mismas personas que ejercen dicha actividad.

Esta segunda característica marca la diferencia entre la autorregulación y la regulación legal y, según Mijatovic, supone una de sus grandes ventajas a la hora de establecer normas de conducta para el periodismo digital, dado que "la autorregulación se erige como la solución para aumentar el control de la actividad online pero ofreciendo al mismo tiempo una mayor flexibilidad que la posible regulación por parte del Estado" $(2013,5)$.

La autorregulación se materializa en una serie de mecanismos entre los que destacan los códigos deontológicos, documentos que definen las expectativas mínimas de la actividad moral, las normas ideales de conducta y una serie de pautas de comportamiento comúnmente aceptadas (Elliott-Boyle, 1985). Por tanto, los códigos recogen los principios que, de acuerdo a su conciencia ética, han de cumplir los periodistas en el cumplimiento de su labor. A pesar de la controversia que estos códigos han generado en algunas ocasiones, todavía son mayoría los profesionales de la comunicación y académicos que los reclaman, basándose en su gran utilidad (Heinonen, 2004).

Hace 20 años, Tina Laitila (1995) realizó un análisis de 30 códigos europeos de ética periodística y comprobó que la mayoría de ellos -21- se habían adoptado o revisado en los años 1990. Según ella, la entonces incipiente introducción de nuevas tecnologías de la información y los cambios políticos, como la caída del muro de Berlín o el proceso de integración europea, fueron algunas de las principales razones para la revitalización del debate sobre la ética periodística que se produjo en esos años.

Por lo tanto, teniendo en cuenta que Internet ha tenido un enorme impacto en el trabajo de los periodistas, sería interesante saber si un debate similar ha surgido de nuevo y si estos cambios se han traducido en nuevas 
directrices éticas. No podemos olvidar que el periodismo ha sido una de las profesiones más cuestionadas en términos éticos en los últimos años y que, como señala Mamonova (2013), Internet es una de las áreas además de prensa, radio y televisión - cuya actividad controlan la mayoría de consejos de prensa europeos.

En Estados Unidos se propuso un nuevo código ético ya a finales de los 90 que abordase estas cuestiones. La idea partió de un grupo de trabajo impulsado por ASNE (American Society of Newspaper Editors) y el Instituto Poynter y el documento hacía referencia a los siguientes aspectos (Mann, 1998):

1. La fiabilidad de los contenidos en línea;

2. El uso de la información contenida en las bases de datos;

\section{Los enlaces;}

4. El control editorial de los contenidos potencialmente dañinos o nocivos;

5. La integridad periodística frente a las presiones comerciales

Por tanto, a raíz de esta situación cabe plantearse: ¿hasta qué punto se han adaptado los contenidos de los códigos deontológicos de los países pertenecientes al Consejo de Europa al nuevo escenario digital? Se trata de una cuestión que ha sido ampliamente estudiada enfocada en escenarios concretos, por ejemplo en Estados Unidos (Whitehouse, 2010), Holanda (Deuze and Yeshua, 2001) España (Ruiz, Masip, Micó, 2007) o en estudios comparativos entre dos países (Micó et al, 2008), pero sobre la que no existen hasta el momento investigaciones que abarquen Europa íntegramente, a diferencia de lo que sí ha sucedido con los consejos de prensa, cuyo estado actual y transformaciones tras la aparición de Internet sí han sido estudiados más en profundidad (Eberwein et al, 2011; Hulin \& Stone, 2013).

El trabajo relativo a los códigos probablemente más ambicioso de todos los que se han llevado a cabo hasta la fecha ha sido el de González Esteban et al (2011) referido a Austria, Alemania, Dinamarca, Estonia, España, Francia y Polonia, y que estudió también otros mecanismos como consejos de prensa y la figura del ombudsman. La conclusión a la que llegan estos estudios es que en la mayoría de estos países no existe ningún tipo de mecanismo de autorregulación del periodismo online de carácter general, y las iniciativas surgidas o bien tienen carácter individual o bien están protagonizadas por medios de comunicación concretos.

Sin embargo, no se ha realizado hasta el momento ningún estudio que analice de manera sistemática cuál es el grado de adaptación de los códigos periodísticos europeos a la nueva realidad del periodismo digital. Por ello, este análisis busca conocer hasta qué punto los códigos nacionales de ética periodística se han adaptado al nuevo entorno online. Para alcanzar este objetivo, se formulan las siguientes tres preguntas de investigación:

PI 1. ¿Son los códigos de más reciente elaboración o actualización los que más aspectos relacionados con Internet incorporan en su articulado?

Como señaló Laitila (1995), la introducción de las nuevas tecnologías fue uno de los motivos que impulsó una actualización o reformulación de los códigos éticos europeos. Se pretende comprobar si el desarrollo continuado de las TIC también ha impulsado o ha sido considerado en las actualizaciones de los códigos durante la última década.

PI 2. ¿Hay alguna zona geográfica de Europa concreta que destaque por un mayor grado de adaptación de sus códigos a Internet y las TIC?

La idea de agrupar a los países por áreas geográficas en función de las semejanzas o puntos comunes entre los que conforman cada uno de los bloques se ha utilizado en estudios comparativos sobre el periodismo en diferentes niveles: como los relativos al grado de profesionalización (Weaver, 1998); al proceso de formación del periodista (Gaunt, 1992; Fröhlich y Holtz-Bacha, 2003); o a la ética periodística (Christians y Traber, 1997; Díaz del Campo, 2012).

Así por ejemplo, Gaunt (1992) agrupa a los países europeos en los siguientes bloques: Grandes potencias 
(Alemania, Francia y Reino Unido), Sur de Europa (España, Grecia, Italia y Portugal), Norte de Europa (Dinamarca, Finlandia, Noruega, Suecia), Europa Central (Bélgica, Holanda, etc.), Europa del Este (Hungría, Polonia, Rumania, etc.) y un quinto bloque con los países más pequeños (Irlanda, Luxemburgo, etc.). El presente análisis también tratará de determinar si alguno de estos bloques ha destacado sobre los demás a la hora de incorporar aspectos relacionados con las TIC a los códigos deontológicos.

PI 3. ¿Qué aspectos propios del periodismo y la comunicación digital se han incorporado a los códigos nacionales.

Esta pregunta trata de identificar las facetas y cuestiones propias del periodismo digital que han sido objeto de regulación en los distintos códigos. Se trata de conocer los aspectos que han despertado mayor interés por ser reguladas abordadas por estos códigos.

\section{METODOLOGÍA}

Para alcanzar los objetivos señalados se identifican cuántos y qué códigos incluyen normas relativas a Internet y a las prácticas periodísticas en este medio. Además, se comprueba cuáles son los aspectos del periodismo digital regulados en esos códigos y en qué sentido se orienta esta normativa.

En concreto, se estudian 45 códigos de ética periodística vigentes actualmente en Europa. Para ello se ha utilizado la base de datos EthicNet[1], elaborada por el Departamento de Periodismo y Comunicación de Masas de la Universidad de Tampere (Finlandia) y en la que todos los textos de los códigos se han traducido al inglés.

En la muestra se han incluido únicamente los códigos generalistas y de ámbito nacional, de modo que los códigos regionales o supranacionales que aparecen en Ethicnet han sido descartados. La muestra tampoco incluye códigos temáticos o normas de autorregulación aplicables únicamente a temas o áreas periodísticas concretas, como el código italiano sobre la actividad periodística y la protección de los menores.

Tratando de precisar y delimitar la búsqueda en los códigos analizados se elaboró una lista de términos (ver Tabla 1). Estos términos, basados en otros empleados en estudios previos de naturaleza similar (Deuze y Yeshua, 2001; Hulin y Stone, 2013; Ruiz, Masip, Micó, 2007), se eligieron ya sea porque son empleados como sinónimos de la expresión "periodismo digital" (p. ej. "periodismo online" o "ciberperiodismo"), porque hacen referencia a algunas de las características y herramientas de Internet más habituales (web, página web, correo electrónico) o a alguno de los servicios web y redes sociales más populares (Twitter, Facebook, etc.).

\begin{tabular}{|l|l|}
\hline Blog & Chat \\
\hline Cyber & Database \\
\hline Digital & Email \\
\hline Facebook & Forum \\
\hline Infographics & Interactive \\
\hline Internet & Link \\
\hline Online & Site \\
\hline Social media & Social networking sites \\
\hline Twitter & Website \\
\hline
\end{tabular}

Tabla 1. Términos de búsqueda empleados para localizar las referencias a aspectos del periodismo digital en los códigos analizados

\section{RESULTADOS Y DISCUSIÓN}

Los resultados obtenidos muestran que sólo un $17,77 \%$ de los códigos estudiados incluyen algún tipo de norma o criterio relativo al periodismo digital, es decir, sólo 8 de los 45 códigos europeos analizados, incluyen referencias a Internet y/o a las tecnologías de la información y la comunicación (TIC). En concreto, se trata de los códigos de Bosnia-Herzegovina, Hungría, Luxemburgo, Noruega, Países Bajos, Polonia, Reino Unido y Rumanía. Por el contrario, no se ha encontrado ni una sola referencia al periodismo digital en los 37 códigos restantes. 
Al mismo tiempo, de esos ocho códigos, tan solo uno dedica un apartado específico al periodismo y los medios digitales: el de Luxemburgo. El resto encuadra diferentes aspectos del periodismo y la actividad online en epígrafes dedicados a los grandes principios de la deontología profesional, como el respeto a la privacidad o la búsqueda de rigor.

Los aspectos vinculados al periodismo digital que aparecen principalmente en los códigos analizados son la gestión del contenido generado por el usuario, el uso de redes sociales como fuente y los enlaces a otras webs.

A continuación se señalan las principales tendencias encontradas en estos códigos.

\subsection{Principios generales aplicados al ámbito online}

Cinco de los ocho códigos que hablan de las nuevas formas de comunicación (Hungría, Países Bajos, Polonia, Reino Unido y Rumanía) mencionan de manera explícita a Internet como uno de los medios que regulan, estableciendo que el material publicado online disfruta de idénticos derechos y debe seguir las mismas obligaciones que el que se publique en los medios tradicionales. Así por ejemplo, el Código de Conducta de Reino Unido señala que "los directores y editores serán los responsables de aplicar el Código al material aparecido tanto en la versión impresa como en la versión digital de la publicación".

De manera similar, los principios relativos a la privacidad de las comunicaciones del código británico recuerdan que "la prensa debe evitar la obtención o publicación de material conseguido mediante el uso de cámaras ocultas o dispositivos de escucha clandestinos, o a través de la interceptación de llamadas realizadas por teléfono móvil, mensajes o correos electrónicos de carácter privado".

La mención a Internet como un medio de masas sometido a los derechos y obligaciones éticas recogidas en el código constituye la única referencia al ámbito online en tres códigos: Hungría, Polonia y Rumanía. Este hecho implica, por un lado, la asunción de que todos los principios y reglas genéricas relativas a los medios tradicionales se pueden adaptar directamente a los medios digitales. Por otro lado, y en parte como consecuencia, en estos países no se ha considerado necesario crear nuevas reglas dirigidas de modo específico al periodismo digital. Por tanto, los periodistas de estos tres países que trabajan en medios digitales deben regirse por los mismos principios éticos que sus colegas de los medios tradicionales.

Mientras, otros códigos como el noruego carecen de una declaración que acoja concretamente a los medios digitales bajo su jurisdicción, pero establecen normas sobre aspectos específicos del periodismo online, como se detallará más adelante.

\subsection{Enlaces}

El uso de hipervínculos es otra de las características propias de los medios digitales y como tal, es recogida por los códigos de Luxemburgo y Noruega. Ambos recogen la advertencia de que las webs de destino de estos enlaces pueden carecer de fiabilidad o de respeto a las normas éticas. En este sentido, el código noruego alerta de que "los enlaces contenidos en una publicación online pueden conducir al lector a otros sitios web o medios electrónicos que no cumplen el contenido del Código Ético" y considera necesario informar al usuario de los enlaces que le dirigen a otros medios.

El código de Luxemburgo, por su parte, establece la obligación del periodista de comprobar que el sitio de destino no alberga material ilícito: "Antes de crear hipervínculos, los medios se comprometen a verificar que los sitios web en cuestión no contienen material ilícito. Si éste fuera el caso, el medio deberá abstenerse de crear cualquier tipo de enlace".

\subsection{Contenidos creados por el usuario}

Internet ha ido facilitando la inclusión del público en el proceso comunicativo, de modo que el contenido generado por el usuario es cada vez más relevante. Históricamente uno de los primeros mecanismos que permitía la participación del público en las publicaciones digitales fueron los comentarios de los lectores. El código holandés incluye en este sentido una sección completa que denomina "respuestas en los sitios web". Según este código, la responsabilidad última del contenido que aparece en la página web del medio 
corresponde al editor. Especifica que aunque "no se puede esperar que todas las respuestas sean examinadas previamente a su publicación", sí que puede decidir "eliminar ciertas respuestas una vez publicadas". Es decir, no considera necesaria la moderación previa de comentarios pero sí la moderación posterior. El código pone especial énfasis en que "si la respuesta a un artículo en el sitio web contiene una grave acusación o una expresión difamatoria hacia una o más personas conocidas, la redacción, a petición de la persona (o personas) en cuestión debe investigar si existen motivos reales para la denuncia o alegación y, si este no es el caso, eliminar esa respuesta".

Para facilitar la labor del medio en este sentido el código holandés recomienda que la web "publique los términos y condiciones para la selección y la publicación de las respuestas". Adoptando esta precaución orienta a los usuarios del tipo de mensajes que podrán tolerarse y de aquellos que no pasarán el filtro de las normas éticas, por lo que podrán ser eliminados de la web.

La facultad y responsabilidad de eliminar contenido de la que goza el medio también está reconocida por el código noruego al hablar de otra herramienta de participación de la audiencia: el chat o encuentro digital. Según este código, una de las responsabilidades más importantes del personal de la redacción respecto a esta modalidad de entrevista consiste en "eliminar de modo instantáneo aquellas intervenciones que no respecten el Código Ético".

\subsection{Otros aspectos}

La posibilidad de modificar los datos disponibles de manera pública online afecta, entre otros aspectos, a la fiabilidad de las fuentes de información y herramientas de consulta accesibles para los periodistas, según recoge el código holandés. En sus propias palabras: "Internet, y los motores de búsqueda asociados a la red, han incrementado notablemente la posibilidad de acceder a los archivos contenidos en bases de datos". Este código da preferencia a la consulta de bases de datos cuyos archivos no pueden ser editados por terceros al contrario de lo que ocurre con el modelo de la Wikipedia, en el que cualquier usuario puede alterar o modificar los artículos que la componen. En términos del código holandés: "En principio, el interés público en archivos fiables, cuyo contenido no se puede alterar, está por encima del posible interés individual por borrar o darle carácter anónimo a la información contenida en esos archivos, cuyos contenidos podrían ser desagradables para esa persona. Este principio tan importante desde el punto de vista social puede ser pasado por alto por razones de interés privado solo en casos excepcionales".

Otra cuestión relativa a la privacidad es la obtención y uso de información personal de los usuarios, generalmente a través de cookies o bien por otras vías. El código noruego es el único que hace referencia a este asunto, considerando como una práctica positiva el "informar a los usuarios de servicios interactivos acerca de los términos en los que quedan registrados y de la posibilidad de explotar el uso que hagan de esos". No declara ilegítima la recolección de esos datos pero recomienda que se informe al visitante de la web de qué información se está registrando y con qué fines se va a utilizar.

Por su parte, el código de Bosnia concibe Internet como una herramienta adicional para promover la comunicación entre los medios y su público, ya que recomienda que "todos los ejemplares de cualquier publicación deberán contener en un lugar apropiado el nombre, dirección, teléfono, y si está disponible, el número de fax, la dirección de la página web y de correo electrónico del editor y de una persona responsable a la que poder dirigir las posibles quejas".

Por ultimo, el código de Luxemburgo se refiere a Internet como un vehículo para promover la difusión del propio código que "será objeto de una publicación específica que aparecerá en el sitio web del Consejo de la Prensa. Lo mismo sucederá en caso de una actualización del Código Deontológico".

\section{CONCLUSIONES}

La presente investigación muestra que el hecho de haber sido adoptado o revisado recientemente no constituye ningún tipo de garantía para que un código de ética periodística incluya referencias al periodismo digital. Así lo prueba el hecho de que 25 de los 45 códigos analizados hayan sido redactados o actualizados desde el año 2000, pero sólo 8 de ellos hayan incorporado cuestiones referidas específicamente a Internet y las TIC. En 
otras palabras, hay 17 códigos que han sido aprobados o actualizados en el siglo XXI pero que no han considerado necesario realizar menciones específicas al periodismo digital. Sin embargo, hay otra realidad igualmente contrastada: muchos de los países con mayor tradición de autorregulación, como Francia o los países nórdicos con excepción de Noruega, no se han decidido a modificar sus códigos para adaptarlos a Internet y las TIC. Entre los países cuyos códigos incluyen más referencias a Internet destacan Países Bajos y Noruega.

En este sentido, Europa del Este es el área geográfica en la que un mayor número de códigos han sido actualizados para incluir aspectos relacionados con Internet y las TIC. Así lo atestiguan los casos de BosniaHerzegovina, Hungría, Polonia y Rumanía. Junto a ellos, nos encontramos con una de las que Gaunt (1992) llama "grandes potencias", como Reino Unido, dos países de Europa Central como Países Bajos y Luxemburgo (aunque éste también se encuadra en la categoría "países pequeños de Gaunt), y uno del Norte de Europa como Noruega.

En definitiva la actitud de los códigos éticos analizados hacia Internet y el periodismo digital se puede caracterizar por un desinterés generalizado y por la falta de homogeneidad. Como ya se ha señalado la mayor parte de los nueve códigos que incluyen referencias a las TICS se limitan a enunciar que el periodismo online está sujeto a los mismos principios que el periodismo tradicional. No existen tendencias homogéneas a la hora de incorporar aspectos específicos del periodismo digital a los códigos éticos. En cada país se incorporan recomendaciones y normas aisladas, fruto de la particularidad de cada contexto nacional, y apenas se encuentran temas y referentes compartidos en estos códigos (ver Tabla 2).

\begin{tabular}{|l|c|c|c|c|}
\hline & $\begin{array}{c}\text { Principios } \\
\text { generales }^{3}\end{array}$ & Enlaces & $\begin{array}{c}\text { Contenidos } \\
\text { usuario }\end{array}$ & Otros \\
\hline Bosnia & $\mathrm{x}$ & & & $\mathrm{x}$ \\
\hline Hungria & & $\mathrm{x}$ & & $\mathrm{x}$ \\
\hline Luxemburgo & $\mathrm{x}$ & $\mathrm{x}$ & $\mathrm{x}$ & $\mathrm{x}$ \\
\hline Noruega & $\mathrm{x}$ & & $\mathrm{x}$ & $\mathrm{x}$ \\
\hline Paises Bajos & $\mathrm{x}$ & & & \\
\hline Polonia & $\mathrm{x}$ & & & \\
\hline Reino Unido & & & & \\
\hline Rumania & & & & \\
\hline
\end{tabular}

Tabla 2. Aspectos del periodismo digital recogidos por los códigos europeos

En este sentido, parece claro que añadir el término "online" o "digital" a principios genéricos de la ética periodística no soluciona todos los dilemas éticos ocasionados por este nuevo escenario para el periodismo. Siguiendo el caso del Código de Conducta de Reino Unido, éste establece al hablar de la privacidad que "toda persona tiene derecho a que se respete su vida privada y familiar, así como lo relativo a su hogar, su salud y su correspondencia, incluyendo las comunicaciones digitales". Sin embargo cabría realizar algunas preguntas: ¿el uso de redes sociales entraría dentro de esa categoría amplia e indefinida de "comunicaciones digitales"? ¿Hasta qué punto puede usar un periodista los mensajes publicados en una red social por un personaje público o anónimo? Es un síntoma de que este nuevo paradigma ofrece problemas específicos que no pueden solucionarse únicamente añadiendo adjetivos a principios éticos tradicionales ya existentes.

Por tanto, resulta razonable plantear la necesidad de una revisión en profundidad del contenido de muchos de estos códigos, tal y como sucedió hace 20 años (Laitila, 1995) y de manera similar al proyecto emprendido hace algunos años en EEUU por The Pew Project for Excellence in Journalism (Kovach y Rosenstiel, 2003) cuando a partir del testimonio de más de 300 profesionales y de la celebración de varios foros públicos, se intentó redefinir cuáles eran los principios básicos ya existentes sobre los que debía fundamentarse un ejercicio periodístico ético y de calidad. En aquel momento fueron la concentración empresarial y el sensacionalismo en los contenidos los dos motivos principales que llevaron a la necesidad de reformular las líneas básicas de consenso.

Y es que, si bien es verdad que en muchos de estos países se han redactado documentos específicos o guías 
referidos al periodismo digital o a algún aspecto concreto de éste (blogs, redes sociales, etc.), los códigos, que al fin y al cabo son el documento máximo de referencia de autorregulación, deberían ser igualmente reformados. De hecho, así sucedió por ejemplo en los años 90, cuando proliferaron las recomendaciones formuladas por asociaciones y consejos de prensa para regular cuestiones muy concretas como la emisión de contenidos sensacionalistas o el tratamiento informativo de la infancia, por mencionar dos ejemplos. Sin embargo, ello no excluyó que los códigos correspondientes fueran reformados en consecuencia (Laitila, 1995). De modo simétrico parece lógico que en la actualidad, con el desarrollo y consolidación de Internet y las TICs, suceda algo parecido y se revise la redacción de los códigos a la hora de abordar cuestiones como por ejemplo la privacidad, cuyo tratamiento, sin tener en cuenta las nuevas tecnologías, ha quedado obsoleta.

En ese sentido, tomando como referencia los criterios marcados por ASNE y The Poynter Institute (Mann, 1998), señalados al inicio de este trabajo, se concluye que su aplicación por parte de las organizaciones correspondientes a la hora de adaptar los códigos de ética periodística ha sido desigual, con referencias, aunque minoritarias, a cuatro de los grandes principios establecidos, la fiabilidad de los contenidos en línea, los enlaces, el control editorial de los contenidos potencialmente dañinos o nocivos y el uso de la información contenida en las bases de datos; y prácticamente ninguna referencia al quinto: la integridad periodística frente a las presiones comerciales.

Por tanto, ésta podrían ser dos de las líneas en las que podrían trabajar las organizaciones profesionales y asociaciones de periodistas europeos a la hora de afrontar la necesaria actualización de contenidos de sus códigos éticos. Se trata de documentos que, como hemos visto, establecen los criterios morales de referencia para todos los periodistas pero que, al mismo tiempo, marcan de manera clara y definida de cara al público cuáles son las reglas del juego de la profesión. Una profesión que difícilmente resultará creíble si esas normas básicas están claramente desfasadas y corresponden a una realidad ya obsoleta.

De manera complementaria también debe señalarse que en los más de 15 años transcurridos desde que se enunciaron estas recomendaciones los códigos periodísticos que sí se han actualizado han incluido aspectos de las TICs no contemplados por el Poynter Institute. Es el caso -por citar solo un ejemplo- del uso de la información registrada sobre los usuarios recolectada por los distintos sitios web. El ritmo de desarrollo de nuevos servicios y funcionalidades dibuja una realidad cambiante que altera incluso principios básicos de la ética tradicional como el respeto a la privacidad en el ya citado caso de las redes sociales, que suponen un cambio significativo en los conceptos tradicionales de público o privado.

En definitiva, las organizaciones de periodistas europeas deberían tratar de adaptarse en la medida de lo posible a ese ritmo tan cambiante y actualizar los contenidos y el funcionamiento de los distintos mecanismos de autorregulación a esta nueva realidad. Quizá, otra medida que posiblemente resultase muy útil en esta línea sería revisar también el Código Europeo de Deontología del Periodismo, aprobado por el Consejo de Europa en 1993). Su hipotética revisión podría servir como acicate para que los distintos países que forman parte de dicha organización llevaran a cabo una revisión similar de forma individual.

\section{REFERENCIAS BIBLIOGRÁFICAS Y HEMEROGRÁFICAS}

ALMIRÓN, N. (2006). "Los valores del periodismo en la convergencia digital: civic journalism y quinto poder". Revista Latina de Comunicación Social, vol. 61. [Consulta: 06/10/2013] http://www.ull.es/publicaciones/latina/200609 Almiron.pdf.

AZNAR, H. (2005): Comunicación responsable. $2^{\mathrm{a}}$ ed. Barcelona: Ariel

BARDOEL, J.; D’HAENENS, L. (2004): “Media Responsibility and accountability: New conceptualizations and practices". Communications, vol. 29, pp. 5-25.

BIRSEN, H. (2011): "Internet Journalism and Journalistic Ethics: Working Conditions and Qualifications of Journalists in the New Media". Journal of US-China Public Administration, vol. 8, n² 2, pp. 230-240.

BOLER, M. (2008): Digital media and democracy. Tactics in hard times. Cambridge: The MIT press. 
BOWMAN, S.; WILLIS, C. (2003): We Media: How audiences are shaping the future of news and information. Reston, Va: The Media Center at the American Press Institute. Hypergene. [Consulta: 08/10/2013] http://www.hypergene.net/

CHRISTIANS, C. y TRABER, M. (1997): Communication ethics and universal values. London: Sage.

CRUZ-ÁLVAREZ, Jesús y SUÁREZ-VILLEGAS, Juan Carlos (2012): “Ética de la participación ciudadana en los discursos periodísticos digitales". El profesional de la información, vol. 21, nº 4, pp. 375-380.

DEMIR, M. (2011): "Importance of Ethic, Credibility and Reliability in Online Journalism". European Journal of Social Sciences, vol. 24, n 4, pp. 537-545.

DEUZE, M.; YESHUA, D. (2001): "Online Journalists Face new Ethical Dilemmas. Lessons from the Netherlands”. Journal of Mass Media Ethics. vol. 16, n 4, pp. 273-292.

DÍAZ DEL CAMPO, J. (2021): "Objetivos pedagógicos básicos en la enseñanza de la ética de la comunicación”. Vivat Academia, n 121, pp. 1-16. [Consulta: 04/10/2013]

http://www.vivatacademia.net/index.php/vivat/article/view/15/16

EBERWEIN, T. et al (Eds.) (2011): Mapping Media Accountability - in Europe and Beyond. Colonia: Harlem.

ELLIOTT-BOYLE, D. (1985): "A conceptual analysis of ethics codes". Journal of Mass Media Ethics, vol. 1, n 1, pp. 22-26.

EVERS, H. (2001): New Moral Dilemmas in Online. Journalism.forummedienethik, vol. 1, pp. 37-46.

FRÖHLICH, Romy y HOLTZ-BACHA, C. (eds.) (2003): Journalism education in Europe and North America: an international comparison. Cresskill, NJ : Hampton Press

GARCÍA CAPILLA, D. J. (2012): "From Postmodern Ethics to the New Ethics of the Me Generation: The Transition from Mass Media to the Internet". Comunicación y Sociedad, vol. 25, nº 1, pp. 165-187.

GAUNT, P. (1992): Making the Newsmakers. International Handbook on Journalism Training. London, Greenwood Press.

GONZÁLEZ ESTEBAN, J.L. et al. (2011): "Professional self-regulation facing new journalistic challenges: a comparative European study”. Revista Latina de Comunicación Social, vol. 66, pp. 426-453.

HAYES, A. S.; SINGER, J. B.; CEPPOS, J. (2007): "Shifting roles, enduring values: The credible journalist in a digital age". Journal of Mass Media Ethics, vol. 22, n 4, pp. 262-279.

HEINONEN, A. (2004): "Journalistic ethics in the age of net". En: SALAVERRÍA, R.; SÁDABA, C. (Eds.): Towards New Media Paradigms. Content, Producers, Organisations and Audiences. Pamplona: Eunate, pp. 213-224.

HOLT, K.; VON KROGH, T. (2010): "The citizen as media critic in periods of media change". Observatorio. vol. 4, n 4, pp. 287-306. . [Consulta: 04/10/2013] http://obs.obercom.pt/index.php/obs/article/view/432

HULIN, A., STONE. M. (eds.), (2013): The Online Media Self-Regulation Guidebook. Vienna: OSCE Representative on Freedom of the Media.

JENKINS, H. (2006): Convergence culture. Where old and new media collide. New York: New York university press.

KOVACH, B., ROSENSTIEL, T. (2003): The Elements of Journalism. New York: Three Rivers Press.

LAITILA, T. (1995): “Journalistic Codes of Ethics in Europe”. European Journal of Communication. vol. 10, pp. $527-544$ 
LARA, T. (2004): “La nueva esfera pública. Los medios de comunicación como redes sociales. Telos. Vol. 76, pp. 128-131.

LÓPEZ GARCÍA, X. (2005): “El ciberperiodismo cultiva sus señas de identidad”. Ámbitos, n 13-14, pp. 45-58.

MAMONOVA, O. (2013): "Area of activity of modern press councils in Europe. En: HULIN, A., STONE. M. (Eds.). The Online Media Self-Regulation Guidebook. Vienna: OSCE Representative on Freedom of the Media, pp. 104-105.

MANN, F. (1998). “New Media” Bring a New Set of Problems . [Consulta: 04/10/2013]

http://www.poynter.org/uncategorized/1734/new-media-bring-a-new-set-of-problems/

MCBRIDE, K. y ROSENSTIEL, T. (2013). The New Ethics of Journalism : Principles for the 21st Century. Thousand Oaks, California: CQ Press.

MICÓ, J,-L. et al (2008): "La ética en el ejercicio del periodismo: credibilidad y autorregulación en la era del periodismo en internet”. Estudos em comunicação, n 4, pp. 15-39. [Consulta: 02/10/2013]

http://www.ec.ubi.pt/ec/04/pdf/02-Jose-Lluis-Mico-La-etica-en-el-ejercicio-del-periodismo.pdf

MIJATOVIC, D. (2013): "Foreword". En: HULIN, A., STONE. M. (Eds.). The Online Media Self-Regulation Guidebook. Vienna: OSCE Representative on Freedom of the Media, pp. 5-6.

PAVLIK, J. (2001): Journalism and New Media. New York: Columbia University Press.

RUIZ, C.; MASIP. P.; MICÓ, J. L. (2007): "Una ética del ciberespacio o una ética en el ciberespacio? Análisis de los códigos de autoregulación de la profesión periodística". En: VV. AA. La ética y el derecho de la información en los tiempos del postperiodismo. Valencia: Fundación COSO, pp. 509-529.

SCHULTZ, T. (2000): "Mass media and the concept of interactivity: an exploratory study of online forums and reader email". Media Culture Society, $\mathrm{n}^{\circ} 22$, pp. 205-221.

WEAVER, David H. (ed.) (1998): The Global Journalist: News People around the World. Cresskill, New Jersey: Hampton Press.

WHITEHOUSE, G. (2010): "Newsgathering and Privacy: Expanding Ethics Codes to Reflect Change in the Digital Media Age". Journal of Mass Media Ethics, vol. 25, nº 4, pp. 310-27.

\section{ANEXO: Lista de códigos}




\begin{tabular}{|c|c|c|}
\hline Pais & Código & $\begin{array}{l}\text { Adoptado/ } \\
\text { revisado } \\
\text { por última } \\
\text { vez en }\end{array}$ \\
\hline Albania & Código de Etica de los Medios de Comunicación de Albania & 2006 \\
\hline Alemania & Código de Prensa de Alemania & 2006 \\
\hline Armenia & Código de los Miembros del Club de Prensa de Ereván & 2002 \\
\hline Austria & Código de Etica de la Prensa de Austria & 1983 \\
\hline Azerbayán & Código de Etica Profesional de los Periodistas & 2002 \\
\hline Bélgica & Código de Pripcipias Reriedistices. & 1982 \\
\hline Bielorrusia & Código de Etica de los Periodistas & 1995 \\
\hline $\begin{array}{l}\text { Bosnia- } \\
\text { Herzegovina }\end{array}$ & Código de Prensa de Bosnia-Herzegovina & 2006 \\
\hline Bulgania & Código Etico de los Medios de Comunicación de Bulgaria & 2004 \\
\hline Ccroacia & Código de Honor de los Periodistas de Croacia & 2006 \\
\hline Chipce. & Código de Recomendaciones Prácticas de los Periodistas & 1997 \\
\hline Dinamarca & Código Nacional de Coaducta & 1992 \\
\hline Esloxaguia. & Código de Etica del Sindicato de Periodistas Eslovacos & 1990 \\
\hline Esloxenia & Código de Etica de los Periodistas Eslovenos & 2002 \\
\hline Espapa & Código Deontológico de la Profesión Periodistica & 1993 \\
\hline Estonia & Código de Etica de la Prensa de Estonia & 1997 \\
\hline Einlandia & Respmendaciones para los Reriodistas. & $2005^{4}$ \\
\hline Erancia & $\begin{array}{l}\text { Carta de los Deberes Profesionales de los Periodistas } \\
\text { Franceses }\end{array}$ & 1938 \\
\hline Georgia & Código de Etica,Periodistica & 2001 \\
\hline Grecia & Código de Etica de los Periodistas Profesionales & 1998 \\
\hline Hungria & $\begin{array}{l}\text { Código Etico de la Asociación Nacional de Periodistas } \\
\text { Húngaros }\end{array}$ & 2007 \\
\hline Irlanda & Código de Conducta & 2007 \\
\hline Islandia & Reglas de Etica para el Periodismo & 1991 \\
\hline Italia & Carta de los Deberes de los Periodistas & 1993 \\
\hline Kosovo & Código de la Prensa de Kosovo & 2005 \\
\hline Letepia. & Código Etiog & 1992 \\
\hline Lituania & Código de Etica de los Periodistas y Editores Lituanos & 2005 \\
\hline Luxemburge & Código Degotológica & 2004 \\
\hline Macedonia & Erincipios de Conducta. & 2001 \\
\hline
\end{tabular}

\begin{tabular}{|c|c|c|}
\hline Malta & Código de Etica,Periodistica & 2000 \\
\hline Moldavia & Código de Etica Profesional de los Periodistas & 1999 \\
\hline Montenegro & Código de los Periodistas de Montenegro & 2002 \\
\hline Decuega & Código de Etica de la Prensa de Noruega & 2007 \\
\hline Raises Baios. & Recomendaciones del Consejo de Prensa de Holanda & 2008 \\
\hline Eolenia & Código de Etica Periodistica & 2001 \\
\hline Portugal & Código de Etica de los Periodistas & 1993 \\
\hline Reine Unide. & Código de Condusta. & 2007 \\
\hline Regública Cbecka. & Código de Etica de los Periodistas & 1999 \\
\hline Rumania. & Código de Etica de los Periodistas & 2004 \\
\hline Rusia & Código de Etica Profesional del Periodista Ruso & 1994 \\
\hline Serbia & Código de los Reriodistas. & 2006 \\
\hline Suesia & Código de Etica para la Prensa, Radio y Televisión & 2006 \\
\hline Suiza. & Declaración de Derechos y Deberes del Periodista & 1999 \\
\hline Тuсquja & Código de Etica Profesional de la Prensa & 1989 \\
\hline Ucrania & Código de Etica de los Periodistas de Ucrania & 2002 \\
\hline
\end{tabular}

\section{Breve semblanza de los autores}


Jesús Díaz-Campo es doctor en Comunicación por la Universidad Complutense de Madrid. Actualmente es profesor de la Facultad de Comunicación y Empresa y miembro del Grupo de Investigación "Comunicación y Sociedad Digital" de la Universidad Internacional de La Rioja. Sus líneas de investigación se centran en la ética de la comunicación, el periodismo digital y la enseñanza del periodismo.

Francisco Segado-Boj es doctor en Comunicación por la Universidad Complutense de Madrid. En la actualidad coordina el Programa de Doctorado en Ciencias Sociales de UNIR y dirige el Grupo de Investigación "Comunicación y Sociedad Digital". Su investigación se centra en los medios audiovisuales (especialmente televisión y humor gráfico) y en los medios digitales.

(1) Esta investigación ha sido realizada en el marco del Gdl-02 "Comunicación y Sociedad Digital”, cofinanciado por UNIR Research (http://research.unir.net).

(2) Ver http://ethicnet.uta.fi/ethicnet_collection_of_codes_of_journalism_ethics_in_europe

(3) Con este apartado se hace referencia a los principios éticos no exclusivos de los medios digitales, sino que se pueden aplicar a cualquier tipo de medio, con independencia de su soporte, como el respeto a la verdad, la presunción de inocencia, el secreto profesional o el respeto a la intimidad y a la vida privada, entre otros.

Ámbitos. Revista Internacional de Comunicación, n.26, año 2014, tercer trimestre (otoño).

Recibido: $1 / 4 / 2014$

Aprobado: 6/6/2014 